\title{
TOURAINE: LE SUJET CONTRE LE SYSTÈME
}

\author{
François Dubet \\ é diretor de estudos na École des Hautes Études en Sciences Sociales. Paris, França. E-mail: \\ <francois.dubet@u-bordeaux.fr> \\ Orcid: 0000-0003-0497-8965 \\ http://dx.doi.org/10.1590/0102-015035/106
}

Il ne m'est pas facile d'écrire sur Touraine car c'est aussi écrire sur mon propre travail sociologique et, plus largement, sur une partie de ma vie. En effet, quand j'ai rencontré Touraine en 1976, il m'a associé au programme de travail qu'il montait alors; nous avons fondé un centre de recherche, le CADIS, et depuis nos chemins n'ont pas cessés de se croiser. Je suis trop proche de Touraine pour en être l'analyste objectif; en revanche, je connais bien l'œuvre et un peu l'homme.

J'essaierai donc de présenter le travail sociologique de Touraine en distinguant plusieurs périodes, même si Touraine lui-même aurait du mal à accepter cette manière de faire tant il est convaincu de n'avoir poursuivi qu'un seul projet. Je distinguerai trois grandes périodes: celle de la société post-industrielle, celle des nouveaux mouvements sociaux et celle de la sociologie du sujet. Ce faisant, je laisserai un peu de côté les livres que Touraine a consacré à l'Amérique Latine et à la vie politique française. Puis, je m'interrogerai sur la nature de l'influence de Touraine dans la vie scientifique et intellectuelle. 


\section{Action et société post-industrielle}

Touraine est entré dans la sociologie dans les années cinquante, au moment où la France se reconstruisait autour de l'industrie. La sociologie aussi se reconstruisait, après la longue éclipse du durkheimisme, autour de deux grandes figures, celle de Raymond Aron et celle de Georges Friedman. Touraine rejoint le groupe de Friedman avec Michel Crozier, Henri Mendras, Jean-Daniel Reynaud, René Tréanton, et prend en charge la question du travail industriel. Son enquête sur la conscience ouvrière aux usines Renault se distingue très sensiblement des deux conceptions dominantes de la conscience de classe. La première est la conception communiste, très largement hégémonique en France et chez les intellectuels: la conscience de classe est une conscience d'exploitation économique qui s'élève vers la globalité grâce à une conscience politique commandée par l'avant-garde du Parti communiste. La seconde venue des États-Unis est d'abord un sentiment d'inégalité et d'exclusion des processus de négociation. Dans La conscience ouvrière (1966) et dans Sociologie de l'action (1965), Touraine définit la conscience de classe des ouvriers comme la tension entre l'organisation du travail industriel et une revendication d'autonomie professionnelle. C'est au nom de cette autonomie que se structure le conflit de classes opposant les maîtres de l'industrie aux travailleurs qui veulent contrôler le développement industriel.

En Mai 68, la position de Touraine se détache nettement de celle de ses pairs. Même si l'événement mêle plusieurs significations, les sociologues se distinguent assez profondément par le sens qu'ils accordent aux évènements. Morin, y voit surtout une rupture culturelle: Mai 68 rompt avec la vieille culture austère et ascétique des sociétés industrielles et appelle une culture de la liberté individuelle, de la communauté élective, de ce qu'on appellera plus tard les valeurs «post-matérialistes». D'autres sociologues, plus 
classiques, comme Boudon et Bourdieu, voient dans Mai 68 une conséquence de la crise de l'université engendrée par la massification et les risques de déclassement social. Crozier, et, dans une certaine mesure, Aron, y voient la manifestation des blocages politiques et institutionnels d'une société qui se modernise sans parvenir à se réformer. Dans Le communisme utopique (1968), Touraine affirme que Mai 68 est la prémisse d'une autre lutte de classes, celle qui oppose la technocratie aux acteurs sociaux, ouvriers, étudiants, femmes... qui opposent leur autonomie à la gestion scientifique et technique de la société. Pour tous ces sociologues, Mai 68 repose sur une sorte de malentendu, car le mouvement adopte le langage gauchiste, bolchévique et communiste pour parler d'autre chose, de la même manière que la révolution française parlait comme les sénateurs romains et que les bolchéviques russes de 1917 parlaient comme les révolutionnaires français.

Mais, on peut considérer qu'à ce moment là l'espace de la pensée sociologique française est tracé pour quelques années. Les uns pensent en termes de mutations culturelles. D'autres pensent en termes de contradictions du système, d'écart entre les intérêts et les contraintes ou bien de tensions entre la socialisation des individus et leurs conditions sociales. D'autres pensent en termes de transformations politiques et institutionnelles, de bureaucratie et de système politique. Touraine fait l'hypothèse que nous entrons dans une société post-industrielle dans laquelle se forment de nouveaux mouvements sociaux et d'autres formes de domination. C'est la thèse centrale de La société post-industrielle (1969).

Je voudrais souligner que cette thèse place Touraine dans une position très particulière. Alors que beaucoup d'intellectuels voient la société comme un pur système de domination et trouvent ainsi un écho immédiat chez les jeunes sociologues proclamés porte-paroles d'un peuple 
muet (c'est le cas de Bourdieu et de Foucault), Touraine est, à la fois, solidaire des mouvements contestataires, tout en pensant que ces mouvements parlent une langue qui n'est pas la leur. Situé à gauche, Touraine ne parle pas la langue ouvriériste et marxiste de la gauche et pense que la gauche se trompe de langue et d'époque. Il est proche de ce qu'on appelle alors la «deuxième gauche», d'une gauche profondément démocratique qui affirme que le développement de l'autonomie est l'enjeu des luttes sociales, et que le «système» n'est pas tout puissant. Bref, il est de gauche tout en étant hostile au thème d'une rupture radicale conduisant les gauches à se trahir elles-mêmes devant l'épreuve du pouvoir. D'emblée, la position intellectuelle de Touraine me semble être décalée; il est solidaire d'acteurs dont il ne partage pas les manières de penser et d'agir. En ce sens, Touraine est le contraire d'un intellectuel organique prêtant sa voix à une cause et à un mouvement, mais c'est un sociologue engagé par une conviction: la société est le produit de ses conflits et de ses orientations culturelles, de ce qui appelle son historicité.

\section{Les mouvements sociaux et l'intervention sociologique}

Au milieu des années 1970, Touraine publie deux livres essentiels. Production de la société (1973) s'efforce d'analyser la vie sociale à partir des mouvements sociaux essayant de contrôler l'historicité, c'est à dire la manière dont les sociétés agissent sur elles-mêmes. La voix et le regard (1978) propose une méthode d'analyse des mouvements sociaux: l'intervention sociologique. Touraine a constitué une petite équipe (Dubet, Hegedus et Wieviorka) pour mettre en œuvre sur un ensemble de mouvements.

Plutôt que de décrire des mobilisations et d'enregistrer des opinions, nous souhaitions dégager des significations et tester leur consistance auprès des acteurs concernés. D’emblée, Touraine a proposé de travailler avec des 
groupes de militants afin de saisir la dynamique des relations entre diverses tendances et surtout afin d'engager les individus dans un travail de réflexion sur eux-mêmes et sur leur action. Or, le propre des militants et des organisations qui les encadrent est de produire des discours idéologiques fortement structurés et «résistant» fatalement aux interprétations des sociologues. D'ailleurs, la plupart du temps, les sociologues analysent ces discours comme des corpus de textes indépendamment de la façon dont les acteurs leur donnent sens. Pour dégager des niveaux de significations et des logiques d'action, il fallait donc «casser» ces discours et inviter les acteurs à réagir à nos propres interprétations sociologiques.

Touraine a choisi d'étudier ces mouvements en formant des groupes de militants engagés à divers niveaux dans la même lutte. Mais pour que les discours ne se cristallisent pas et ne se referment pas sur eux-mêmes, l'intervention sociologique les soumet à trois grands stimuli. Le premier d'entre eux est le débat interne des militants qui ne peuvent développer leurs orientations sans se heurter à d'autres. Mais ce forum ne suffit pas et les sociologues invitent les militants à discuter avec leurs adversaires. Ainsi se crée et se mesure une distance entre les discours et les rapports sociaux «réels», distance qui conduit les militants à réfléchir sur leur propre action. L'originalité la plus forte de l'intervention sociologique ne tient pas seulement à l'organisation de ces débats et à la durée du travail des groupes - plusieurs dizaines d'heures de discussion -, elle réside dans le rôle même des chercheurs qui analysent les débats et le travail des groupes et proposent aux acteurs leurs propres interprétations. En se plaçant du point de vue de l'hypothèse relative à la formation, ou non, d'un nouveau mouvement social, ils soumettent leurs analyses aux acteurs et leur demandent d'y réagir. Alors que la plupart des manuels de méthodologie insistent sur la neutralité du chercheur identifié à une 
simple machine à enregistrer, la plus discrète possible, ici le chercheur engage une discussion sociologique avec les acteurs à la suite de très longs débats.

Le mot intervention doit être pris dans sons sens le plus fort et il faut bien admettre que cet outil méthodologique a pris à rebrousse-poil quelques habitudes professionnelles soucieuses de «rupture épistémologique» et d'objectivisme. Le chercheur s'engage dans une longue relation avec les acteurs et fait de cette relation l'objet central de son analyse. Bien sûr, cette relation elle-même doit être objectivée, ce qui n'implique pas que l'on objective celui que l'on étudie. Ceci suppose de multiplier les groupes de recherche afin de s'assurer que la stabilité des observations dépasse la singularité des individus et des groupes. Il importe aussi de recomposer les équipes de chercheurs afin que leurs idées ou leur personnalité soient, elles aussi, placées sous contrôle. Quand on obtient les mêmes «faits» à partir de groupes et 20 de chercheurs différents, on peut imaginer que ces «faits» sont suffisamment consistants pour résister aux hypothèses des chercheurs. Sur ce plan, nous ne serons pas déçus car, si nous avons observé des traces de nouveaux mouvements dans les luttes étudiées, nous n'avons pas trouvé le nouveau mouvement que nous espérions. Alors que l'on aurait pu soupçonner l'intervention sociologique d'être une sorte de prédiction créatrice plus ou moins manipulatrice, voire une espèce de thérapie de groupe, l'expérience a plutôt prouvé le contraire. La planète que nous cherchions ne laissait voir au mieux que les traces de quelques astéroïdes.

De 1976 à 1982, Hegedus, Touraine, Wieviorka et moi avons travaillé intensément et parcouru des milliers de kilomètres pour étudier une série de luttes sociales plus ou moins «nouvelles». La sociologie ressemblait à une aventure et je dois à Touraine de l'avoir partagée.

Les luttes étudiantes. A la suite d'une longue mobilisation étudiante en 1976, nous avons formé deux groupes 
de militants. La question était de savoir si ce mouvement étudiant était en mesure de considérer que le savoir et la connaissance pouvaient être enjeux de luttes sociales, si les étudiants pouvaient en appeler à la constitution d'une expérience autonome contre une gestion routinière et bureaucratique de l'université et si, au-delà, ils en appelaient à la création de véritables universités en France, pays où celles-ci ont toujours occupé une place secondaire en dépit de l'explosion de la démographie étudiante. Malgré l'acharnement des chercheurs à défendre cette hypothèse, force a été de constater que les militants de nos groupes étaient plus les témoins d'une crise de l'université que les porteurs d'un véritable projet. Sur cette crise du fonctionnement universitaire, amphis de masse, débouchés flottants, distance aux enseignants, les étudiants projetaient une représentation révolutionnaire et ouvriériste des mouvements sociaux. En fait de nouveau mouvement social, nous avons plutôt observé la crise du système universitaire telle que Mai 68 l'avait révélée. Ni enjeu, ni rapport social autonomes et propres au mouvement étudiant n'ont émergé de cette recherche dont il ne resta parfois que l'amertume des militants dont le sol idéologique se dérobait quand ils observaient, par exemple, qu'ils étaient les meilleurs alliés des enseignants les plus conservateurs et que la production intellectuelle et scientifique se faisait au plus loin d'eux. Depuis cette époque, on ne peut guère dire que cette analyse pessimiste ait été démentie. Au contraire, le sentiment de crise de l'université s'est accentué et la peur de ne pas trouver de place au terme des études s'est accrue aux dépens de luttes appelant à la transformation interne des universités elles-mêmes.

Les luttes anti-nucléaires. Avec le mouvement anti-nucléaire, nous étions a priori bien plus proches de notre hypothèse, car la critique du nucléaire était celle de technostructures largement publiques bien plus que celle du capitalisme proprement dit. Par ailleurs, la défense de la 
nature ou, plus exactement, de sa maîtrise par les citoyens était de toute évidence un enjeu lourd et promis à prendre de plus en plus d'ampleur. Les groupes que nous avons formés confirmèrent ces hypothèses. Mais ils montrèrent en même temps qu'il y a loin de ces principes à la formation d'un mouvement organisé. L'écologie radicale portée par des communautés exemplaires invitait bien des militants à rejeter la science plutôt qu'à la faire entrer dans le jeu démocratique, tandis que d'autres combattaient l'Etat plus qu'une nouvelle classe dirigeante. Il se créait aussi une grande distance entre les résistances locales contre l'implantation d'un équipement et d'une industrie, et un mouvement de contestation générale. Quarante ans plus tard, les choses n'ont guère changées: la conscience et la sensibilité écologiques se sont imposées dans un grand nombre de pays sans que se forme pour autant un mouvement social à la mesure de ces enjeux majeurs. A l'exception de l'Allemagne des années quatre-vingt où les Verts étaient plus pacifistes qu'écologistes, les mouvements écologiques ne sont pas à la taille de ces défis. Ils sont dispersés dans une multitude d'organisations, de groupes et d'ONG eux-mêmes déchirés entre fondamentalistes et politiques et peut-être avons-nous trop crû que les luttes écologiques naissantes des années soixante-dix referaient à leur manière le chemin accompli par le mouvement ouvrier un siècle auparavant.

Lutte régionaliste. Dans les années 1970, une nouvelle génération de militants régionalistes voulait rompre avec la défense traditionnelle des langues régionales en liant cette affirmation culturelle à des luttes économiques contre la fermeture des usines et surtout avec les luttes des viticulteurs du Midi de la France, en Occitanie. Ce qu'il pouvait y avoir de nouveau dans cette mobilisation, c'était la volonté d'en appeler au développement contre le système formé par l'alliance du centralisme et des notables locaux. Une identité culturelle pouvait devenir agent de développement et de 
conflit, comme c'était le cas, sous une forme démocratique, en Catalogne et, sous une forme bien plus violente, au pays basque espagnol. La défense de la langue et celle de l'économie se sont croisées mais ne se sont jamais véritablement articulées. Les viticulteurs étaient moins des régionalistes que des défenseurs déçus de l'Etat national qui semblait les abandonner. Depuis lors, l'Etat s'est décentralisé, mais le rêve du mouvement n'existe plus guère. Les luttes économiques et les défenses culturelles ont repris chacune leur route alors que ce type de revendication ne subsiste, comme en Corse, que là où il se noue autour du nationalisme. Mais il serait bien difficile de parler de nouveaux mouvements sociaux dans ce cas.

$\mathrm{Si}$, à la manière de Popper, on définit une démarche scientifique par le fait que des hypothèses sont «falsifiables», nos études sur les nouveaux mouvements sociaux sont plutôt rassurantes: nous n'avons pas véritablement trouvé les mouvements que nous cherchions. En tous cas, nous ne les avons pas trouvés sous la forme que nous attendions. Mais si l'on pense aussi qu'une bonne recherche doit permettre d'anticiper sur des évolutions, sinon sur des événements, là aussi, l'histoire des mouvements que nous avons étudiés avec tant de soin et d'énergie, n'a pas totalement démenti nos conclusions d'alors. Les nouveaux mouvements, on peut aujourd'hui penser aux mobilisations altermondialistes, sont sans doute dispersés dans un ensemble de tendances et de logiques allant de la critique antisystème aux mouvements de contre-expertise sans que leur point d'équilibre ne se stabilise jamais vraiment et sans qu'ils trouvent une expression politique capable de s'imposer. Là encore, ce jugement sociologique n'est en rien une mesure du poids historique de ces luttes et moins encore un jugement moral. Ces mouvements sont plus éclatés et plus radicaux que nous le pensions alors, en même temps que leurs demandes sont plus rapidement institutionnalisées. Au fond, beaucoup d'entre 
eux ont fini par imposer leurs questions sans se transformer en organisations puissantes et sans que la gauche s'en fasse véritablement le relais politique. L'écologie est reconnue comme un enjeu central, la condition féminine aussi, sans que ces enjeux aient été portés par des mouvements «puissants et organisés», reproduisant un peu l'histoire du mouvement ouvrier qui était plus ou moins consciemment notre référence cachée.

Le syndicalisme ouvrier. Dès l'origine du programme de recherche sur les nouveaux mouvements sociaux, Touraine avait pensé qu'il nous faudrait étudier le mouvement ouvrier auquel il avait consacré près de vingt années de son travail. Bien sûr, il n'était pas question de savoir si ce mouvement devenait un «nouveau mouvement social», mais plutôt de voir comment, il se recomposait dans un contexte économique et social marqué par le déclin de la société industrielle.

24 Dans tous les milieux où nous avons travaillé, les militants avaient le sentiment se heurter à de grandes difficultés et de voir s'affaiblir la conscience de classe ouvrière. Les communautés de classe étaient érodées ou quasiment détruites par la vague de désindustrialisation qui touchait de grands secteurs. Des militants se plaignaient du déclin des valeurs industrielles, de la disparition des «modèles» communistes et, surtout, d'une transformation du capitalisme éloignant le «vrai» patron de la direction de l'entreprise. Nostalgie parce que, même si cet univers s'épuisait, la condition ouvrière restait difficile, d'autant plus difficile même que les ouvriers étaient les premières victimes du chômage de masse qui s'installait durablement en France. Les syndicalistes se sentaient enfermés dans des actions défensives et dans un fractionnement croissant de la classe ouvrière.

Solidarité. Au mois d'août 1981, démarre aux chantiers navals de Gdansk ce qui sera sans doute une des plus grandes grèves de l'histoire. Cette grève s'étend dans toute 
la Pologne, le parti communiste est obligé de négocier avec Solidarité et seul le coup d'Etat de décembre 1982 réprimera le mouvement pour offrir quelques années de survie au régime. Wieviorka et moi sommes partis en Pologne en janvier 1982 pour y monter, avec l'aide de sociologues polonais et francophones, une intervention sociologique dans trois grandes villes: Gdansk, Katowice et Varsovie. Du point de vue sociologique, les choses étaient relativement claires. Les militants ouvriers étaient portés par trois logiques fortement intégrées. D'abord une conscience ouvrière s'opposant aux dirigeants des entreprises au nom des conditions de travail et des salaires. Ensuite, une conscience démocratique en appelant à l'ouverture d'un espace public et institutionnel permettant aux demandes sociales de s'y exprimer. Enfin, une conscience nationale combattant des dirigeants identifiés à la domination soviétique. Fortement agrégées, ces revendications ont fini par incarner la société contre un pouvoir de plus en plus illégitime et vide. L'intervention sociologique a aussi montré qu'au fil des mois ces trois formes de conscience tendaient à se distinguer et à se séparer. La conclusion de cette enquête allait de soi: nous assistions à la fin de la société communiste; une société entière l'avait rejetée et il suffira que l'Empire s'écroule pour qu'il n'en reste rien. En même temps, on voyait bien que les forces du nationalisme populiste et religieux et celle de la démocratie libérale allaient se disputer la définition de la nouvelle société. La mort du totalitarisme et le triomphe de l'économie de marché n'étaient certainement pas la fin de l'histoire.

On ne peut penser que la vie sociale est une pure construction subjective et que, dans ce cas, les sociologues ne peuvent que rendre compte de la manière dont les acteurs eux-mêmes rendent compte de cette réalité. A l'opposé, on ne peut croire que ces acteurs sont de simples supports de positions sociales dont les discours et les pratiques 
ne seraient que des conséquences mécaniques et programmées. Si la compréhension et l'explication sont bien deux opérations intellectuelles différentes, il faut bien admettre qu'elles peuvent être associées si l'on pense que les acteurs agissent, réfléchissent et s'interrogent sur les mécanismes qui les déterminent. Et comme leurs questions ne sont pas très différentes de celles que posent les sociologues, même quand ils n'en adoptent pas le vocabulaire, la sociologie de Touraine peut être définie comme une forme particulière et particulièrement contrôlée de débat.

\section{La fin des sociétés}

Au terme de cette période, les choses semblaient claires aux yeux de Touraine. Le scénario selon lequel un nouveau mouvement social, fut-il difracté en plusieurs luttes, devait succéder au mouvement ouvrier comme le mouvement central de la société post-industrielle n'était pas tenable. Autant il était sûr que nous assistions au Le retour de l'acteur (1984), autant cela ne pouvait pas signifier que cet acteur serait incarné par un mouvement social homogène. D'une part, nous sommes engagés dans un long processus de déclin de la société industrielle: montée des populismes, fin de l'hégémonie occidentale, multiplication des voies de la modernité, crise des social-démocraties, règne des identités, triomphe du néolibéralisme... D’autre part, c'est l'idée même de société qui se défait sous nos yeux et c'est dans cette voie que s'engage la réflexion de Touraine depuis la fin des années 1980.

Le livre qui marque à mes yeux une certaine rupture, mais qui est aux yeux de Touraine la radicalisation de sa thèse initiale, est sans doute Critique de la modernité (1992). Dans cet ouvrage, Touraine défend la thèse d'une double nature contradictoire de la modernité. D'un côté, à la manière de Weber, la modernité est définie comme le triomphe de la Raison, de la science et de la technique. Mais la modernité 
est aussi le triomphe du sujet, c'est-à-dire de la subjectivité personnelle, des droits humains, de la liberté de conscience, de la foi, de l'authenticité personnelle, de la capacité de se définir soi-même contre les contraintes du fonctionnement de la vie sociale. Ainsi, le sujet se manifeste dans mille expériences et dans mille mouvements dans lesquels les individus n'en appellent pas seulement à leurs intérêts, mais aussi à leur liberté et à leur singularité. Bien sûr, si cet appel repose toujours sur des principes non sociaux, il reste aussi enchâssé dans des conditions culturelles et sociales tout en se définissant comme une volonté d'arrachement à ces conditions. Dans la pratique, les choses sont toujours ambivalentes puisqu'elles conduisent à opposer un être pour soi à un être pour la société: sa sexualité contre les catégories du genre, l'éthique religieuse contre la morale religieuse, l'identité pour soi à l'identité collective imposée. En fait, le sujet se manifeste dans les tensions de son expérience comme Touraine le montre dans deux ouvrages: Pouvons-nous vivre ensemble? (1997) et Le monde des femmes (2006). L'exercice est d'autant plus difficile que la scène sociale est aujourd'hui dominée par des mouvements, des anti-mouvements, qui en appellent à la défense des identités collectives, des autorités religieuses, des États forts et de la violence. Face à eux, il n'est pas certain que le «patriotisme constitutionnel» défendu par Habermas suffise à protéger les démocraties.

Avec La fin des sociétés (2013), et Nous, sujets humains (2015), la pensée de Touraine prend une inflexion plus radicale. Si on définit l'idée de société par l'intégration des subjectivités, des cultures et des systèmes sociaux, et au niveau des individus, par l'intégration des statuts, des rôles et des personnalités, ce qui correspond à la conception de Durkheim, de Parsons mais aussi de Bourdieu, alors nous ne vivons plus dans cette forme de société. Non seulement cette forme de société est en crise, comme le montrent les crises des institutions - écoles, familles, églises - et celles 
des démocraties accusées d'être, à la fois, inefficaces et peu représentatives, mais cette crise est bien plus profonde. En effet, si on définit une société par l'intégration d'une économie nationale dirigée par une bourgeoisie et un État nationaux, par une culture nationale et par un système politique et institutionnel souverain, alors, nous sommes au-delà des sociétés avec des souverainetés affaiblies, les coexistences plus ou moins pacifiques des cultures et des identités et, surtout, le monde des réseaux et la domination d'une économie financière internationale détachée de l'économie et des rapports sociaux réels. Au vieux conflit de classes et aux mouvements sociaux classiques, se juxtapose le face-à-face entre un système non social et des acteurs qui se définissent comme des sujets au-delà et contre le système. Écartons un malentendu: la fin des sociétés n'est pas la fin du social, car la formation des sujets et des mouvements d'opposition et de résistance est toujours enracinée dans des conditions sociales, des modèles culturels et des cadres institutionnels et politiques. Mais le social ne prend plus la forme de la société, de l'intégration de l'acteur et du système et du conflit située dans cette intégration et visant son renforcement.

\section{Un rayonnement plus qu'une École}

J'aimerais me livrer à un exercice s'apparentant à une intervention sociologique dans laquelle le chercheur essaie d'expliquer aux acteurs le sens de leur action après les avoir longtemps lus et écoutés. Tout le risque de cette tentative vient de ce que l'acteur en question est ici l'œuvre de Touraine, de ce que son «analyste» est un de ses élèves littéralement fabriqué par Touraine et, plus encore, un élève qui lui en rend grâce. Si Touraine ne m'avait pris sous son aile voici quarante ans, je ne serais certainement pas sociologue aujourd'hui, tant les autres manières d'être sociologue me semblaient ennuyeuses, à la fois rhétoriques et loin de la vie sociale. 
La situation de Touraine est paradoxale. Dès les années soixante, il est un sociologue et un intellectuel célèbre, ne partageant cette position qu'avec moins d'une demi-douzaine de ses collègues français, et encore en comptant large. Il est aussi connu et largement reconnu dans la plupart des pays du monde. Il est invité partout, écrit dans tous les grands journaux et toutes les grandes revues, ses ouvrages sont tous traduits et il n'y a pas de doute sur sa place dans l'histoire intellectuelle du demi siècle qui vient de s'écouler. Beaucoup plus qu'il ne le croit souvent, Touraine est admiré, tenu pour un grand parmi les grands et ce vaste réseau de lecteurs, d'étudiants et de collègues déborde très largement les sentiments d'admiration impressionnée qu'il engendre auprès de tous ceux qui se sentent toujours très en dessous du niveau auquel se situe son œuvre. Il est vrai que Touraine a souvent l'élégance de vous laisser croire que ce que vous faites est intéressant, parfois intelligent, alors même qu'il suffit de lire la plupart de ses livres pour être sans illusion sur son propre travail.

Le paradoxe vient de ce que cette reconnaissance, cette admiration et ces amitiés n'ont pas fait de Touraine le maître d'une Ecole sociologique dont nous pourrions compter les disciples et mesurer leur degré de fidélité et de conformisme à la pensée du père fondateur. En France, il n'y pas l'École de Touraine, comme il y celle de Boudon et de Bourdieu, comme il y a eu celle de Crozier, comme se forme une école pragmatique ou ethnométhodologique. Les tourainiens sont inspirés par Touraine, nourris de Touraine, ce ne sont pas des gens qui imitent le maître, répandent sa pensée, contrôlent des revues... Ils se sentent les membres d'un collège invisible, pas les élèves d'une salle de classe ou les soldats d'un régiment.

Ecartons immédiatement une explication invraisemblable, celle qui tiendrait à une certaine «faiblesse de caractère» de Touraine, à une nonchalance ou au fait que son œuvre naviguerait dans le mainstream inodore et incolore de 
la sociologie. En revanche, je crois pouvoir dire que Touraine n'a peut être jamais eu véritablement envie de faire une École. Ceci le distingue très nettement de Durkheim bien sûr, mais aussi plus près de nous, de Boudon et de Bourdieu dans le cas français. Car c'est un vrai travail que de faire une École. Il faut sélectionner des élèves, en faire des disciples, s'assurer de leur conformisme et de leur fidélité, exiger d'être le centre de leurs références, interdire de citer et d'évoquer les «ennemis», sauf pour les assassiner... Il faut aussi contrôler des places, des postes, des éditeurs, des revues, placer ses gens afin que l'enthousiasme admiratif des élèves se conjugue à leurs intérêts de carrière bien compris. Il faut enseigner et placer des élèves à l'École normale supérieure et à Sciences-Po afin de s'attacher les futures élites. Je n'aurai pas la cruauté d'évoquer ceux qui ont fait École, à commencer par Durkheim pour citer un collègue qui ne fâche plus, mais c'est un vrai travail que de faire une École, c'est une stratégie de tous les instants, un jeu de marquages et de démarquages, une vigilance constante dans un monde intellectuel perçu comme un champ de bataille et une guerre plus ou moins chaude. Il faut créer ou prendre une revue, y placer des amis qui sont aussi des vassaux, s'assurer que tous les textes contribuent à votre gloire, écarter les fâcheux, tenir les brides serrées... Bref c'est une «besogne» et une affaire de caractère. Or Touraine a consacré son travail à autre chose et je ne suis pas sûr que celui que l'on perçoit parfois comme un peu cassant ait jamais consenti à devenir un chef d'École, pour ne pas dire de secte. Pour des raisons que j'ignore, mais qui me semblent a priori bonnes, Touraine préfère les bandes amicales aux bataillons disciplinés. En tous cas, après des années de travail commun et malgré des différences de position et de renommée considérables entre nous, je peux témoigner que si Touraine faisait peser des exigences de travail relativement lourdes sur ses compagnons, il ne leur demandait jamais d'être «dans la ligne» de sa pensée. 
On pourrait attribuer cette influence libérale à un trait de caractère, mais au fond, ceci me semble anecdotique et «contingent». Car si Touraine a choisi l'influence plutôt que l'École, c'est parce que son style intellectuel ne se prête guère à la formation d'une doctrine aisément imitable et reproductible. La pensée de Touraine est construite sur une série de décalages et de porte-à-faux qui fascinent ses lecteurs et ses collaborateurs et dissuadent la plupart de l'imiter. Cette pensée ne se résume pas en quelques recettes élémentaires simples et facilement réductibles aux formules sommaires qui font le bonheur des manuels scolaires et des étudiants. Plus encore, il construit des concepts qui ressemblent à ceux que l'on utilise de manière banale pour, en réalité, dire autre chose, à commencer par ceux de mouvement social ou de sujet.

Quand, dans les années 1950 et 1960, le monde intellectuel français était encore largement sous influence communiste, marxiste et fonctionnaliste américaine, Touraine étudiait la conscience ouvrière comme une figure de l'expérience de travail, et non pas comme une conscience politique ou comme une forme de «clairvoyance historique», d'adhésion à un sens de l'histoire déjà là. Quand il étudie les mouvements sociaux, il donne au concept un sens si singulier que la plupart des mouvements sociaux, surtout les «nouveaux mouvements», apparaissent loin du compte. De la même manière, ce qu'il définit comme l'historicité est le contraire de l'historicisme; Un Désir d'histoire (1977) est plus un désir d'action, qu'une volonté de faire l'histoire. Dès la fin des années 1980, ce sociologue met en cause l'idée même de société. Ce qui est un comble! Ce style intellectuel ouvre des portes, fait tomber des barrières et des blocages, donne des idées nouvelles, libère l'imagination de nombreux chercheurs, mais il n'est pas le mieux armé pour fonder une École. «Pire», au fil de son histoire intellectuelle, Touraine a presque toujours existé seul contre les Écoles qui 
dominaient alors. Ecrire Sociologie de l'action (1965) en pleine mode structuraliste ou Production de la société (1973) dans le climat structuro-marxiste des années soixante-dix n’est pas la manière la plus sûre d'être dans l'air du temps.

\section{Un engagement décalé}

Comme Touraine est un intellectuel engagé autant qu'un sociologue, son engagement est lui-même des plus décalés. Homme de gauche, il n'a cessé de critiquer la gauche et de se démarquer intellectuellement des mouvements qu'il soutenait politiquement. Le sociologue du mouvement ouvrier critique le syndicalisme dominant sous la coupe du Parti communiste quand la plupart faisaient allégeance. Du côté des étudiants en Mai 68, il défend le mouvement contre ses propres idéologies. Du côté de l'Unité populaire au Chili, totalement solidaire des victimes de Pinochet, Touraine ne partage pas les idéaux et les imaginaires politiques d'une grande partie des militants de l'Unité Populaire. Ami des sociologues latino américains, il n'en partage pas les théories de la dépendance - Vie et mort du Chili populaire (1973); La parole et le sang (1988). Il étudie, avec quelques autres, les nouveaux mouvements sociaux qu'il appelle de ses voux, tout en démontrant qu'ils sont extrêmement faibles et englués dans d'anciennes idéologies. Il soutient activement la gauche française tout en écrivant un ouvrage expliquant que son succès ouvrira L'après socialisme (1980). Il n'y a peut-être que dans la Pologne de 1980 que l'histoire et la sociologie se sont réconciliées. Sur la plupart de ces dossiers, Touraine a eu raison; le tout politique a affaibli le mouvement ouvrier, le gauchisme n’est pas ce qui reste de Mai 68, les mythes révolutionnaires d'Amérique latine ont fait des héros, des martyrs et des guérillas, pas des révolutions. Quand à la victoire de la gauche française en 1981, on aurait du mal à y voir une mutation socialiste sous le seul prétexte que c'est un épisode important de l'histoire du parti socialiste. L'analyse sociologique 
des grèves de 1995 est au plus loin de celles qui voient un mouvement social dans tout ce qui bouge. Suivre Touraine sur tous ces points n'était pas toujours facile, c'était même la façon la plus sûre de se fâcher avec ses amis, et ce n'est certainement pas comme cela que l'on fait École car les écoliers aiment bien adhérer.

En fait, tous ces décalages conduisent à penser que l'œuvre de Touraine s'est toujours construite sur trois plans relativement distincts. Touraine est un théoricien de la sociologie construisant des concepts analytiques organisant une représentation de la vie sociale; c'est à la fois un penseur de l'action, un penseur du système et un penseur du sujet, que celui-ci soit conçu comme un sujet historique ou comme un sujet individuel. Touraine est aussi un historien, ce qui le distingue de nombre de ses collègues théoriciens, car il pense en termes de phases, de périodes et de types de société. Enfin, Touraine est un penseur politique, un penseur de la conjoncture et des rapports de force. Il est comme Parsons tout en étant hostiles aux cathédrales théoriques, comme Weber tout en ne s'abandonnant pas aux délices des grandes fresques historiques, et comme Marx tout en ne réduisant pas la vie sociale à la domination. C'est pour cette raison qu'il a tant d'influence, souvent tant de séduction, et qu'il «épuise» tous ceux qui voudraient l'imiter comme des élèves imitent un modèle. Le style intellectuel de Touraine n'est pas confortable, pour lui-même probablement, et plus encore pour les autres. L'hypothèse sociologique, l'hypothèse historique et l'hypothèse politique ne sont pas forcément harmonieuses, parce que le monde est ainsi fait, et l'on peut affirmer parfois qu'un mouvement social est sociologiquement fondamental, historiquement secondaire et politiquement sans effet. Ou le contraire. Mais cet inconfort participe de la fascination admirative que provoque une pensée toujours renouvelée, le «dernier Touraine» surprend toujours, alors même qu'elle ne cesse de tisser la même trame... 
Rien ne montre mieux ces décalages et ces paradoxes que la méthode d'intervention sociologique. Traditionnellement, on considère qu'une bonne méthode est celle qui évite que le chercheur intervienne sur son objet. Personne n'est choqué par la vieille habitude faisant qu'un chercheur observe un acteur du dehors tout en affirmant de façon routinière que cet acteur est un acteur, voire un sujet. Or, Touraine propose «d'intervenir», c'est-à-dire de confronter les acteurs aux hypothèses des chercheurs afin de voir ce qu'ils en font. Ce qui est la position la moins naturelle et la moins confortable qui soit et, bien évidemment, ce qui laisse planer un soupçon de manipulation des acteurs par les chercheurs. Après tout, chacun pourrait avoir envie d'être prophète. Mais le chercheur qui aura abandonné le confort de l'observation «objective» pour les charmes du prophétisme sera profondément déçu: dans la plupart des interventions sociolo34 giques, les hypothèses tourainiennes les plus optimistes ne sont pas vérifiées. La production de connaissances résulte d'un échec de la «prophétie» issue des hypothèses construites du point de vue d'un mouvement social et d'un sujet. Ainsi, Touraine invite-t-il à changer de posture de travail et de recherche, tout en privant le chercheur des conforts croisés du positivisme et de la prophétie. Bien que la méthode d'intervention sociologique ait été reprise et développée, force est de reconnaître qu'elle prend à rebrousse poil les habitudes professionnelles les mieux établies et qu'elle ne peut s'exporter comme une technique statistique.

\section{$* * *$}

Il n'est donc pas étonnant que Touraine ait construit une œuvre fondamentale et reconnue comme telle, qu'il ait une influence bien plus grande qu'il ne croît lui-même, que nombre de ses collègues l'aiment et l'admirent. Si Touraine rayonne, il n'a pas fait une Ecole. Il est bien plus proche de 
Weber que de Durkheim, de Wright Mills que de Parsons, et après tout, c'est assez flatteur. J'espère que Touraine me pardonnera l'impertinence de ces propos. D'abord parce qu'ils participent de l'amitié que je lui porte, ensuite parce qu'il en a toujours plus appelé à la liberté de ses élèves qu'à leur soumission et à leur obéissance.

\section{François Dubet}

é sociólogo, professor emérito da Universidade de Bordeaux e diretor de estudos na École des Hautes Études en Sciences Sociales, em Paris. Pesquisou sobre movimentos sociais, juventude, educação e sobre as desigualdades sociais e os sentimentos de justiça.

\section{Bibliographie}

Dubet, François et al. 1978. Lutte étudiante. Paris: Seuil.

Dubet, François et al. 1980. La prophétie antinucléaire. Paris: Seuil.

Dubet, François et al. 1981. Le pays contre l'État. Paris: Seuil.

Dubet, François et al. 1982. Solidarité. Paris: Fayard.

Dubet, François et al. 1984. Le mouvement ouvriers. Paris: Fayard.

Touraine, Alain. 1965. Sociologie de l'action. Paris: Seuil.

Touraine, Alain. 1966. La conscience ouvrière. Paris: Seuil.

Touraine, Alain. 1968. Le mouvement de Mai ou le communisme utopique.

Paris: Seuil.

Touraine, Alain. 1969. La société post-industrielle. Paris: Denoël.

Touraine, Alain. 1973. Production de la société. Paris: Seuil.

Touraine, Alain. 1973. Vie et mort du Chili populaire. Paris: Seuil.

Touraine, Alain. 1977. Un désir d'histoire. Paris: Stock.

Touraine, Alain. 1978. La voix et le regard. Paris: Seuil.

Touraine, Alain. 1980. L'après socialisme. Paris: Grasset.

Touraine, Alain. 1984. Le retour de l'acteur. Paris: Fayard.

Touraine, Alain. 1988. La parole et le sang. Paris: Odile Jacob.

Touraine, Alain. 1992. Critique de la modernité. Paris: Fayard.

Touraine, Alain. 1997. Pourrons-nous vivre ensemble? Egaux et différents.

Paris: Fayard.

Touraine, Alain. 2006. Le monde des femmes. Paris: Fayard.

Touraine, Alain. 2013. La fin des sociétés. Paris: Seuil.

Touraine, Alain. 2015. Nous, sujets humains. Paris: Seuil. 


\section{TOURAINE: LE SUJET CONTRE LE SYSTEME}

FRANÇOIS DUBET

Résumé: Malgré son ampleur et la multitude des objets qu'elle aborde, l'œuvre de Touraine est conduite par une thèse centrale: la tension entre l'acteur social et le système. Cette idée s'est déployée sur plusieurs thèmes: le travail, la société post-industrielle, les mouvements sociaux et l'idée de sujet. Dans tous les cas, Touraine oppose le sujet, ses droits et sa subjectivité, aux logiques de la domination et du fonctionnement de la société. Intellectuel de gauche, Touraine n'est cependant pas l'intellectuel organique des partis et des mouvements sociaux dont il soutient les combats, car tout son travail scientifique vise à mettre en lumière ce qui oppose le travail du sujet aux idéologies constituées.

Mots-clés: Intervention Sociologique; Mouvement Social; Sujet Historique.

\section{TOURAINE: O SUJEITO CONTRA O SISTEMA}

Resumo: Apesar de sua grande amplitude e multiplicidade dos objetos que aborda, a obra de Touraine é conduzida por uma tese central: a tensão entre o ator social e o sistema. Esta ideia se espalhou por diversos temas: o trabalho, a sociedade pós-industrial, os movimentos sociais e a ideia de sujeito. Em todos os casos, Touraine opõe o sujeito, seus direitos e sua subjetividade às lógicas de dominação e do funcionamento da sociedade. Intelectual de esquerda, Touraine não é, no entanto, o intelectual orgânico dos partidos e movimentos sociais cujas lutas ele apoia, pois todo seu trabalho científico visa destacar o que opõe o trabalho do sujeito a ideologias constituidas.

Palavras-chave: Intervenção Sociológica; Movimento Social; Sujeito Histórico. 


\section{TOURAINE: THE SUBJECT AGAINST THE SYSTEM}

Abstract: Despite its large scope and the multitude of objects it addresses, Touraine's work is driven by a central thesis, i.e. the existing tension between the social actor and the system. He has developed this argument on several themes: work, post-industrial society, social movements and the idea of subject. In all cases, Touraine opposes the subject - its rights and its subjectivity to the logics of domination and of the functioning of society. Being a left-wing intellectual, Touraine, however, is not an organic intellectual of the political parties and social movements that he supports. The whole of his scientific work aims at highlighting the opposition between the subject's work and the established ideologies.

Keywords: Sociological Intervention; Social Movement; Historical Subject.

Recebido: 06/07/2018 Aprovado: 25/02/2019 